\title{
An Efficient Algorithm for Improving Ber and Papr of Ofdm System by Using Nonlinear Companding Transform
}

\author{
A.Vinisha ${ }^{1}$, Kumar.keshamoni $^{2}$, S. Swetha ${ }^{3}$ \\ M.Tech Scholar, ECE Aurora scientific Technology And Research Academy (ASTRA),Hyderabad,T.S,India \\ Sr.Asst.Prof in Dept of ECE Aurora scientific Technology And Research Academy (ASTRA), Hyderabad, T. S, \\ India
}

\begin{abstract}
One of the challenging issues for OFDM (Orthogonal Frequency Division Multiplexing) systems are its PAPR. Proposed review and analysis of various OFDM PAPR reduction methods are based on computational, complexity, spectral spillage and performance. Our aim is a non linear companding technique is proposed to reduce high PAPR of OFDM. Which can use companding for Peak to Average Power Ration control is explored for linking a nonlinear transmit power amplifier with OFDM. In this article analyses the improvement in Bit Error Rate and overall system performance by employing non linear companding technique for PAPR reduction in Digital Video Broadcasting system.
\end{abstract}

Index-terms: Bit Error Rate (BER), DVB-T, OFDM, PAPR.

\section{Introduction}

In remote and wire-line communication Orthogonal Frequency-DivisionMultiplexing is a multicarrier transmission scheme that has become the technology of choice for next generation system, due to its high speed data rates, quality service, efficiency, robustness against narrow band interference and frequency selective fading. These carriers (subcarriers) have different frequencies and they are orthogonal to each other. By making the sub-channels the narrowband and individual channel experiences almost no fading, this makes receiver as simple design as possible. Later on, Saltzberg analyzed the OFDM performance and observed that the crosstalk was the severe problem in this system. Although each subcarrier in the principal OFDM systems overlapped with the neighborhood carriers are the orthogonality can still be preserved through the staggered QAM method. However, the difficulty will emerge when a large number of subcarriers are required. Early OFDM applications have number of subcarriers can be chosen up to 34 . Those 34 symbols will be appended with redundancy of a guard time interval to eliminate inter-symbol interference (ISI). In the 1990s, OFDM systems have been exploited for high data rate communications. IEEE 802.11 standard the carrier frequency can go up as high as $2.4 \mathrm{GHz}$ to $5 \mathrm{GHz}$. The developments of Researchers tend to pursue OFDM operating at even much higher frequencies nowadays. The best example is IEEE 802.16 standard proposes yet higher carrier frequencies ranging from $10 \mathrm{GHz}$ to $60 \mathrm{GHz}$.

Today OFDM used in many wireless standards such as terrestrial digital video broadcasting (DVB-T), digital audio broadcasting (DAB-T), and adopted in wireless local area networks (WLANs) (IEEE 802.11a, ETSI Hiperlan2) and wireless metropolitan area networks (IEEE 802.16d). The main drawback of OFDM is its high (PAPR) which causes serious degradation in performance when nonlinear power amplifier (PA) is used. This high PAPR forces the transmit PA to have a large input back off (IBO) in order to ensure linear amplification of the signal, which significantly reduces the efficiency of the amplifier, Furthermore, high PAPR requires high resolution for the receiver analog-to-digital converter (A/D). Since the dynamic range of the signal is much larger for high PAPR, a high-resolution quantizer is required to reduce quantization error, which requires more bits and places a complexity and power burden on the receiver front end Many PAPR reduction schemes based on different techniques, such shaping, block coding, partial transmit sequence (PTS) technique and selective mapping (SLM) technique, phase optimization, tone reservation and injection and non linear companding transform schemes have been proposed in literature [4]. In this paper, we proposed and analyse non linear companding technique to reduce the PAPR of OFDM signal. Non linear companding has better system analysis including Peak to Average Power reduction and Bit Error Rate, the implementation complexity is less and no bandwidth expansion.

\section{Related Work}

The most attractive schemes are nonlinear transform due to the reason it's a good system performance including PAPR reduction and BER. The implementation is complex and no expansion of bandwidth. In the stage of nonlinear companding transform is the $\mu$-law companding which are based on the audio or video processing algorithm low and then shown optimal performance than compare to clipping methods, $\mu$-law mainly focuses on enlarging signals which are very less amplitude and keep peak signals are unchanged thus it can 
improve the peak to average power of the transmitted signals and are possibly results in exceed the saturation region of HPA make system performance worse. Main variation in between clipping and nonlinear Companding transform is described as: 1) Clipping is a method deliberately it clips high signals when the ampitude of the original OFDM signals are higher than of given threshold and then clipped signals cannot be recovered at the destination side. However nonlinear companding transform is compand to original OFDM signals use the strict monotone increase function. Finally the companded transform signals are at the transmitter it can be recovered effectively through the corresponding inversion of nonlinear function at the receiver side 2) Nonlinear companding transform extract small signals while coding a large signals to increase the signals immunity from noisy signals then after while the clipping method does not change small signals in that channel. Therefore clipping method suffers from mainly three problems: In-band distortion, then out-of-band radiation, and peak regrowth after DAC conversion. Based on these results the system performance is degraded due to the clipping it may not be optimistic. In this work to transform the amplitude of the original Orthogonal Frequency Division Multiplexing signals are described into the distribution with its PDF

$\mathrm{F}_{\mathrm{SC}}(\mathrm{s})=\mathrm{k}_{\mathrm{S}}+\mathrm{b} \quad(\mathrm{k}<0, \mathrm{~b}>0)$.

Finally this nonlinear transform function is derived as .

$\mathrm{C}(\mathrm{x})=\sqrt{6 \sigma\left[1-\exp -\left(\frac{x^{2}}{2 \sigma^{2}}\right)\right]}$

This nonlinear companding transform belongs to the exponential companding scheme. Problem design criteria are two types of nonlinear companding transformation which is based on error function and exponential function, respectively, have been proposed. The well-known original signls are highly sharp rectangular-like power spectrum. The well-known property will be affected on PAPR reduction technique, ex. slower spectrum roll-off, side-lobes are more, and higher adjacent channel interference. Lot of PAPR reduction schemes cause spectrum side-lobes getting, but the nonlinear companding transforms cause less side-lobes spectrum. Error and Exponential companding transform have very less impact on original power spectrum differencing to the $\mu$-law companding scheme. The major reason is Error, Exponential companding methods are not only extracting the small amplitude signals but also coding the large amplitude which can maintain the average unchanged average power based on optimal parameters, and it can raise the immunity of small amplitude signals from various noise signals. Therefore $\mu$-law companding transform can increases or maintain reliable power level and therefore it requires linear operation region in HPA.

\section{Proposed Criteria For Papr Reduction In Ofdm Systems}

Most of the existing solutions still have some drawbacks. one is the trade-off between Peak to Average Power reduction and some factors such as bandwidth. Proposed criteria is the Peak to Average power reduction are to find the approach that it can reduce Peak to Average Power largely and at the same time it can keep better performance in terms of the following factors as possible.

$\checkmark$ Capability of PAPR reduction: Primary factor is considered to selecting the PAPR reduction algorithm which has few harmful sides of the effects on such as in-band distortion and out-band radiation.

$\checkmark$ Average Power: This can also reduce the PAPR in OFDM by using mean of the power on original signals to increase a higher linear operation region in High Power Ampilfier and thus resulting in the reduction performance of $\mathrm{BER}$.

$\checkmark$ Implementation complexity: Basically, the complexity of these techniques are exhibits besr capability of PAPR reduction. In this work both delay, and hardware requirements for the PAPR reduction very less.

$\checkmark$ Bandwidth expansion: one of the rare resources in this work is bandwidth. The bandwidth expansion directly results in the data code rate loss due to beside information. Therefore, when channel coding is used the loss in data rate is increased further due to side information. Therefore, the loss in bandwidth due to side information should be avoided.

$\checkmark \quad$ BER performance degradation: Aim of the PAPR reduction is to obtain efficient preformance including Bit Error Rate than that of the original Orthogonal Frequency Division Multiplexing system. All the methods have an improved Bit Error Rate at the receiver it should be paid more attention in practice. However the side information is received in error at the receiver part, which may also result in whole erroneous data frame and thus the Bit Error Rate performance, is reduced.

$\checkmark \quad$ No need additional power: The design of a wireless system should always take into consideration the efficiency of power. If an operation of the technique which reduces the Peak to Average Power needs more additional power, then it degrades the Bit Error Rate performance when the transmitted signals are normalized back to the original power signal. 


\subsection{Performance Analysis}

In this paper we consider a typical model of Orthogonal Frequency Division Multiplexing system with 256 sub-carriers and 16-Quadurature Amplitude Modulation constellation in which oversampled Orthogonal Frequency Division Multiplexing sequences with the oversampling rate of 4 are used to analyze Peak to Average Power Ratio reduction and Bit Error Rate performance based on different schemes as shown in the following fig.1. At preset the clipping levels has been selected to $80 \%$ of the maximum of the original Orthogonal Frequency Division Multiplexing symbols in the clipping scheme and the number of the reserved tone is 20 in Transmit Receive scheme. In this article we used Partial Transmit Ssequence scheme, for that the rotation vectors belong to set $\{+1,-1\}$, and number of sub-blocks is 16 . However we got the $2^{15}$ searches is for each optimal Partial Transmit Sequence. These two schemes are Partial Transmit Sequence and Transmit Receive is used for all the side information has not been submitted to the receiver. We refer the nonlinear companding transform the error companding is that proposed in [11] and the exponential companding is based on [9].

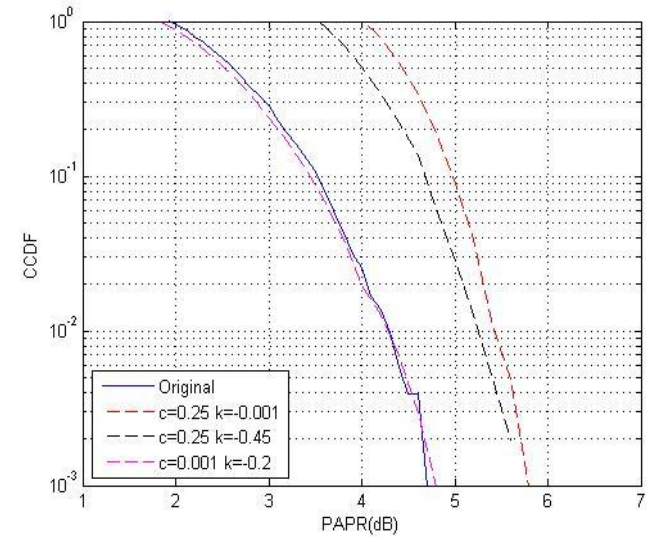

. Fig.1. Comparision of CCDF based on different PAPR reductions

As shown in Fig. 1, different curves of the Complementary Cummulative Distribution Function have been given for random original Orthogonal Frequency Division Multiplexing symbols generated and different Peak to Average Power Ratio reduction schemes. From Fig. 1 it is very clear that all schemes can reduce the Peak to Average Power Ratio largely in Orthogonal Frequency Division Multiplexing system.

Their performances of the Peak to Average Power Ratio reduction are different. For example when various PAPRs are $2 \mathrm{~dB}, 4.3 \mathrm{~dB}, 6.5 \mathrm{~dB}, 6.6 \mathrm{~dB}, 7 \mathrm{~dB}$ and $11.9 \mathrm{~dB}$ for the exponential companding, error companding, Partial Transmit Sequence, TR, clipping scheme and original Orthogonal Frequency Division Multiplexing signals, respectively. Obviously, the signals companded by the nonlinear companding transform with exponential function can reduce the Peak to Average Power Ratio largest and the Peak to Average Power Ratio reduction of the clipping scheme is the smallest among these typical methods. Although clipping scheme can improve its performance of the Peak to Average Power Ratio reduction through reducing its preset clipping level A. However, the performance of the Bit Error Rate will be degraded largely when its preset clipping level is reduced.

\subsection{BER Performance analysis using Nonlinear Companding Transform}

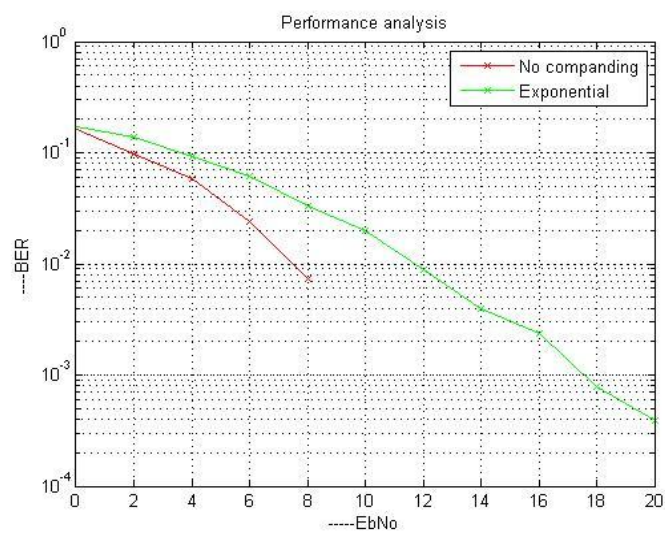

Fig.2. BER Vs SNR 
From fig-2 the SNR of the companded Orthogonal Frequency Division Multiplexing (OFDM) signals obtained by using the Nonlinear Companding Transform is equal to $12 \mathrm{~dB}$ at a Bit Error Rate (BER) of $10^{-0.6}$ for QPSK.

\section{Conclusion}

In this article we have proposed a PAPR reduction scheme to improve the Bit Error Rate performance in OFDM system. PAPR reduction can be reduced by applying non-linear companding technique. This methodology gives $1-1.5 \mathrm{db}$ reduction in PAPR with the comapnding technique is achieved. The simulation results have shown that the proposed Nonlinear Companding Transform algorithm could offer better performance in terms of PAPR reduction without increasing the complexity of the system performance.

\section{References}

[1] T. Hwang, C. Yang, G. Wu, S. Li, and G. Y. Lee, “OFDM and its wireless application: A survey,” IEEE Trans. Veh. Technol., vol. 58, no. 4, pp. 1673-1694, May 2009.

[2] Tao Jiang, Wenbing Yao, Peng Guo, Yonghua Song, and Daiming Qu Two Novel Nonlinear Companding Schemes With Iterative Receiver to Reduce PAPR in Multi-Carrier Modulation Systems IEEE TRANSACTIONS ON BROADCASTING, VOL. 52, NO. 2, JUNE 2006

[3] X.B Wang T.T Tjhung and C. S Ng, - Reduction of peak to average power ratio of OFDM system using companding technique, IEEE Trans broadcasting vol 45, no 3, pp 303-307, sept 1999.

[4] T. Jiang, W. D. Xiang, P. C. Richardson, D. M. Qu, and G. X. Zhu, 一On the nonlinear companding transform for reduction in PAPR of MCM signals,\| IEEE Trans. Wireless communications, vol. 6, no. 6, pp. 2017-2021, Jun. 2007.

[5] H. Nikookar and K. S. Lidsheim, - Random phase updating algorithm for OFDM transmission with low PAPR,\| IEEE Trans. Broadcasting, vol. 48, no. 2, pp. 123-128, Jun. 2002.

[6] T. Jiang and G. X. Zhu, - Nonlinear companding transform for reducing peak-to-average power ratio of OFDM signals,\| IEEE Trans. Broadcasting, vol. 50, no. 3, pp. 342-346, Sept. 2004.

[7] T. Jiang, Y. Yang, and Y. Song, -Exponential companding transform for PAPR reduction in OFDM systems, IEEE Trans. Broadcasting, vol. 51, no. 2, pp. 244-248, Jun. 2005.

[8] T. Jiang, W. Yao, P. Guo, Y. Song, and D. Qu, - Two novel nonlinear companding schemes with iterative receiver to reduce PAPR in multicarrier modulation systems, IIEEE Trans. Broadcasting, vol. 52, no. 2, pp. 268-273, Mar. 2006

[9] H. Nikookar and K. S. Lidsheim, "Random phase updating algorithm for OFDM transmission with low PAPR," IEEE Trans. Broadcasting, vol. 48, no. 2, pp. 123-128, Jun. 2002.

[10] X. B.Wang, T. T. Tjhung, and C. S. Ng, "Reduction of peak-to-average power ratio of OFDM system using A companding technique," IEEE Trans. Broadcasting, vol. 45, no. 3, pp. 303-307, Sept. 1999.

[11] R. O’Neill and L. B. Lopes, “Envelope variations and spectral splatter in clipped multicarrier signals,” in Proc. IEEE PIMRC'95, Toronto, Canada, Sept. 1995, pp. 71-75

\section{About Author Details}

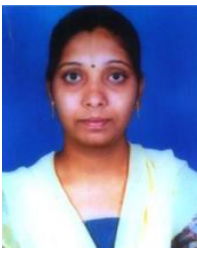

A.Vinisha received B.Tech degree in ECE from Gurunanak Insitute Of Technology,Ibrahimpatnam,R.RDist,T.S. Pursuing M.Tech in ECE From Aurora Scientific Technology And Research (ASTRA),Hyd,T.S

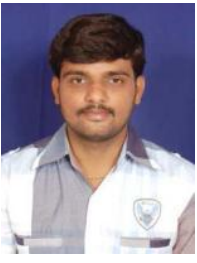

Kumar. Keshamoni obtained his B.Tech in Electronics \& Communication Engineering from the University of JNTU, his M.Tech in VLSI Design from the University of JNTU-Hyderabad. $\mathrm{He}$ is currently the Sr. Asst. Professor in Department of Electronics \& Communication Engineering, Aurora's Scientific, Technological and Reseach Academy, in University of the JNTUH Hyderabad. Kumar. Keshamoni is Chair of World Committees Associate Member of UACEE (Universal Association of Computer \& Electronics Engineers), Member of IEEE Vehicle Community, Member of IAENG and an Advisory Board of WATT (World Association of Technology Teachers). He is also on the Advisory \& Editorial Board of IJETAE, an Associate Editor of IJPRET, Editor of IJOART, Editorial Board \& Review Committee of IJSER, an Editorial \& Review Board of IJTRA, Editor, writer \& Peer Reviewer of edition International Publisher, Peer Reviewer of AJET, Review Committee of IJERT, Editorial \& Review Board of IJOAR, Review Board of IJASCSE, Advisory\& Editorial Board of IJRDET, Editorial Board of IJEERT Member of IAENG, Writer\& Review Board of IJEVS, Advisory Board of WRJET, Scientific Committee \& Editorial Review Board on Engineering and Physical Sciences of WASET and Editorial Board of IAASSE. He is also working as a member for 10 International conference committees. He has published about 20 Research articles in International Journals and 5 papers in National and International Conferences and attended several National, International Conferences, and Workshops \& Faculty Development Programs in different organizations. His recent research interests lie in the area of Embedded, VLSI Design, Digital Signal Processing and Nanotechnology. 


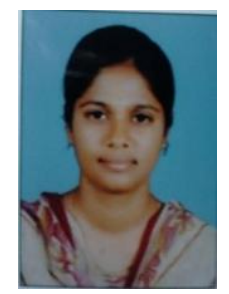

Mrs. S Swetha received M.E degree in Microwaves \& Radar Engg. From OU in 2010, she did B.Tech degree in ECE from JNTUH in 2007.

Presently working as Sr. Assistant professor in ASTRA, Hyderabad. 